\title{
Estudio comparativo sobre las capacidades físicas del adulto mayor
}

DOI: https://doi.org/10.33262/ap.v2i4.13

(c) (1) (9) (2)

Comparative study on the physical capacities of the older adult

Orlando Rodrigo Carrasco Coca., ${ }^{1}$ Mónica Cabezas Cabezas Flores., ${ }^{2}$ Ronal Vinicio Gómez Nicolalde. ${ }^{3} \&$ Xavier Alejandro Zamora Parra. ${ }^{4}$

\begin{abstract}
.
The present work seeks to present a comparative study between two care centers for the elderly, in relation to the deterioration of their physical capacities (sarcopenia), caused by the natural wear and tear of their age, which directly affect their functional capacities, which they grant to the adult greater a quality of life that responds directly to the treatment that is provided on it. A sample of 10 people in an age range between 57 and 88 years was used for assessment by applying physical tests, and 18 people in an age range between 80 and 100 years for assessment by means of a physical activity questionnaire, between the centers: " Mi Amigo Divino Elderly Home "and" San Ignacio de Loyola Senior Adult Home ", through which their physical level was determined through functional tests considering the physical capacities of strength, flexibility and static balance of both the upper and lower body, Functionally assessing the degree of mobility of the elderly, totaling real data of subjects with different characteristics of a social nature, for which a mainly sedentary

${ }^{1}$ Universidad de las Fuerzas Armadas "ESPE”. Carrera de Pedagogía de la Actividad Física y Deportes. Quito, Ecuador.orcarrasco@espe.edu.ec

${ }^{2}$ Universidad de las Fuerzas Armadas "ESPE”. Carrera de Pedagogía de la Actividad Física y Deportes. Quito, Ecuador.mmcabezas@espe.edu.ec

${ }^{3}$ Universidad de las Fuerzas Armadas "ESPE". Carrera de Pedagogía de la Actividad Física y Deportes. Quito, Ecuador.ronaldinho_rem@hotmail.com

${ }^{4}$ Universidad de las Fuerzas Armadas "ESPE”. Carrera de Pedagogía de la Actividad Física y Deportes. Quito, Ecuador. xazamora@espe.edu.ec
\end{abstract}


population is described from a quantitative parameter. The data presented in this research provide a very important reference on the current situation of the elderly in relation to the progressive loss of both muscle volume and strength.

Keywords: Strength, flexibility, balance, physical abilities, elderly, sarcopenia.

\section{Resumen.}

El presente trabajo busca exponer un estudio comparativo entre dos centros de atención al adulto mayor, con relación al deterioro de sus capacidades físicas (sarcopenia), provocada por el desgaste natural de su edad, que afectan directamente en sus capacidades funcionales, que otorgan al adulto mayor una calidad de vida que responde directamente al tratamiento que se brinde sobre ella. Se utilizó una muestra de10 personas en un rango de edad entre 57 y 88 años para valoración mediante aplicación de test físicos, y 18 personas en un rango de edad entre 80 y 100 años para valoración mediante cuestionario de actividad física, entre los centros: "Hogar de Ancianos Mi Amigo Divino" y "Hogar del Adulto Mayor San Ignacio de Loyola", mediante los cuales se determinó su nivel físico a través de pruebas funcionales considerando las capacidades físicas de fuerza, flexibilidad y equilibrio estático tanto del tren superior e inferior, valorando de manera funcional el grado de movilidad del adulto mayor, totalizando datos reales de sujetos con distintas características de índole social, por lo que se describe a una población mayoritariamente sedentaria desde un parámetro cuantitativo. Los datos presentados en esta investigación brindan una referencia de gran importancia sobre la situación actual del adulto mayor en relación a la pérdida progresiva tanto del volumen muscular como de la fuerza de los mismos.

Palabras claves: Fuerza, flexibilidad, equilibrio, capacidades físicas, adulto mayor, sarcopenia.

\section{Introducción.}

El envejecimiento se muestra como un proceso natural en los seres humanos, que trae consigo una serie trasformaciones en el organismo. El deterioro de órganos y sistemas es una de las principales consecuencias de este proceso, al cual se le da el nombre de Sarcopenia; según (Casanueva, Kaufer, Pérez, \& Arroyo, 2008) la sarcopenia es entendida como una alteración progresiva propia del envejecimiento, provocada por una reducción de las reservas de proteína del cuerpo, dónde la atrofia muscular conlleva a una pérdida de la fuerza en conjunto con la disminución de volumen de los mismos, promoviendo una alteración negativa en la movilidad global del adulto mayor. Además siendo un vehículo para el aumento de enfermedades como diabetes y osteoporosis, e incluso alcanzando un deterioro en la tolerancia a la glucosa, todo esto desencadenando consecuencias que otorgan incrementos en las tasas de mortalidad. (Gil, 2010) 
Así, la fuerza y el tamaño muscular en el adulto mayor se ve disminuida progresivamente, tal como también se reconoce en la "teoría del desgaste", dónde se sabe, inicia un proceso de decadencia sobre distintas partes del cuerpo del adulto mayor (Riedel, 2000), cuya principal causa de su acelerada progresión, lo provoca la falta de actividad física. Siendo esta razón, un desencadenante sobre necesidad de inducir al mantenimiento de la motricidad en el adulto mayor, para llegar a mantener y mejorar sus capacidades motoras, mediante la aplicación de actividad física propia para su edad, buscando un fortalecimiento muscular en personas de mediana y avanzada edad con el fin de retardar el envejecimiento y mejorar su calidad de vida.

Está demostrado que las personas que realizan actividad física de manera regular, mejoran sus capacidades motoras, donde el adulto mayor no es una excepción; estos pueden verse beneficiados tras una aplicación controlada y específica según la necesidad que tenga el grupo humano; considerando: El tipo de actividad a ser aplicado, la intensidad del ejercicio, la duración del ejercicio, la frecuencia del ejercicio y el ritmo de progresión del mismo. (Cevallos, 2012), con ejercicios que orienten a facilitar la realización de tareas de la vida cotidiana, dentro del entorno del adulto mayor.

Entendido esto, la forma más adecuada para comenzar un trabajo de recuperación de las capacidades físicas en el adulto mayor, es partir desde una prescripción de ejercicio, para así tener un referente importante al momento de la programación del entrenamiento; teniendo en cuenta según (Maranzano, 2013) la "elaboración de un cuestionario de salud, evaluación del nivel de condición física, elección de los ejercicios, determinación de las cargas de trabajo, (y) planificación del entrenamiento" para su aplicación.

Por lo tanto, la prevención de la pérdida de estas capacidades físicas adquiere una dimensión especial para evitar el deterioro de la calidad de vida y la dependencia de los adultos mayores, volviéndose válido e importante conocer como la incidencia de actividad física actúa positivamente sobre la sarcopenia en el adulto mayor, como coadyuvante en el mejoramiento de su calidad de vida, en conjunto con la alimentación y la medicina (Gil, 2010).

Pero, para llevar a cabo una prescripción de actividad física en el adulto mayor, primero se debe lograr una valoración de las capacidades físicas en el mismo. Volviéndose importante a la par, definir términos relacionados con dicha valoración de las capacidades físicas, tal como el significado de fuerza, entendida como "la capacidad de vencer una resistencia y reaccionar en su contra mediante una tensión muscular" (Cervera, 1999), permitiendo además, el desplazamiento del cuerpo de un lugar a otro, apoyado principalmente del desarrollo del sistema osteomuscular(Durán, 2013). Equilibrio, entendido como la capacidad neuromuscular que "permite conservar y recuperar formas... después de la realización de movimientos" (Martin, 2001). Y flexibilidad, entendida como "aquella amplitud de movimientos a nivel articular" (Heredia, Donate, Medrano, Riera, \& Mata, 2011). Siendo estas tres, las capacidades a evaluarse en test específicos creados para conocer las cualidades 
físicas que poseen los adultos mayores. Y en aquellos casos donde no puedan ejecutar los movimientos que dichos test obligan debido a su complejidad física, la aplicación del "Cuestionario Internacional de Actividad Física" (IPAQ) (Booth, 2000, citado por Smith, 2004) como referente para identificar la condición de los mismos, sin la necesidad de mayor esfuerzo físico.

Así pues, para conocer la condición física de los adultos mayores, previo a la prescripción de actividad física específica, este artículo tendrá como objetivo, valorar a dos grupos de control en un estudio comparativo, a fin de reconocer el nivel de mantenimiento de las capacidades físicas en el adulto mayor.

\section{Material y método.}

Para las tomas, se aplicaron las siguientes pruebas de valoración de condición física, a una muestra de estudio en dos centros de atención al adulto mayor: 6 adultos mayores en un rango de edad entre 73 y 88 años de edad del "Hogar de Ancianos Mi Amigo Divino" y a 4 adultos mayores en un rango de edad entre 57 y 78 años de edad del "Hogar del Adulto Mayor San Ignacio de Loyola":

Chair-sit and reach test (Rikli y Jones, 1999). Se estudiaron 10 sujetos.

Tiene como objetivo valorar la flexibilidad del tren inferior. Se pide al sujeto que se coloque al borde de una silla, con una pierna en flexión, y la otra en extensión con los pies en contacto con el piso. Juntando las dos manos, una sobre la otra, intentará tocar o sobrepasar la línea de la punta del pie de la pierna en extensión con la yema de su dedo más sobresaliente. Se mide la distancia entre la yema del dedo y la punta del pie, una extremidad a la vez.

Back Scratch Test (Rikli y Jones, 1999). Se estudiaron 10 sujetos

Tiene como objetivo valorar la flexibilidad del tren superior. Se pide al sujeto que coloque sus manos detrás del cuerpo y lo más cerca la una de la otra, mediante rotación interna y externa del hombro. Se mide la distancia que existe entre los dedos más sobresalientes de cada mano.

Chair stand test (Rikli y Jones, 1999). Se estudiaron 10 sujetos.

Tiene como objetivo valorar la fuerza del tren inferior. Se pide al sujeto que realice el movimiento de levantarse completamente y sentarse completamente de una silla, las veces que pueda durante treinta segundos, manteniendo sus brazos cruzados frente a su pecho.

Arm curl test (Rikli y Jones, 1999). Se estudiaron 10 sujetos.

Tiene como objetivo valorar la fuerza del tren superior. Se pide al sujeto que tome una mancuerna, de cinco libras para mujeres, y ocho libras para hombres, y que realice la mayor 
cantidad de flexiones y extensiones de codo durante treinta segundos, una extremidad a la vez.

Static Balance test (Rikli y Jones, 1999). Se estudiaron 10 sujetos.

Tiene como objetivo valorar el equilibrio estático. Se pide al sujeto que coloque sus manos en la cadera, flexionando a la vez una rodilla, enviando su pie por detrás de la pierna que se encontrará en apoyo con el piso. Se determinará el tiempo que logren mantener esta posición, una extremidad a la vez.

En los casos cuyo potencial físico no permitió la valoración de capacidades físicas, se evaluó mediante el "Cuestionario Internacional de Actividad Física" (IPAQ) (Booth, 2000, citado por Smith, 2004), que consiste en un determinado número de preguntas dónde se identifica el nivel de actividad física. Del mismo, participaron 11 adultos mayores entre 80 y 100 años de edad del "Hogar de Ancianos Mi Amigo Divino"; y 7 adultos mayores entre 85 y 99 años de edad del "Hogar del Adulto Mayor San Ignacio de Loyola":

Además, se empleó el software SPSS (Statistical Packageforthe Social Sciences) como procesador estadístico de datos.

\section{Resultados test físicos.}

Tabla 1. Hogar del Adulto Mayor San Ignacio de Loyola

\begin{tabular}{|c|c|c|c|c|c|c|}
\hline NOMBRES & EDAD & $\begin{array}{l}\text { FLEXION } \\
\text { CODO }\end{array}$ & SENTADILLA & $\begin{array}{c}\text { EQUILIBRIO } \\
\text { ESTATICO }\end{array}$ & $\begin{array}{l}\text { FLEXIBILIDAD } \\
\text { TREN } \\
\text { SUPERIOR }\end{array}$ & $\begin{array}{l}\text { FLEXIBILIDAD } \\
\text { TREN } \\
\text { INFERIOR }\end{array}$ \\
\hline Cauca Encarnación & 88 & $\begin{array}{l}D=16 \\
I=13\end{array}$ & 11 & $\begin{array}{l}D=0^{\prime \prime} \\
I=0^{\prime \prime}\end{array}$ & -42 & $\begin{aligned} D & =+0,5 \\
I & =-0,5\end{aligned}$ \\
\hline Chagua Marcela & 84 & $\begin{array}{l}D=0 \\
I=0\end{array}$ & 9 & $\begin{array}{c}D=4,96^{\prime \prime} \\
I=2,22^{\prime \prime}\end{array}$ & -11 & $\begin{array}{l}D=+2 \\
I=+3,5\end{array}$ \\
\hline Lliangalí Cuello Lucila & 76 & $\begin{array}{l}D=16 \\
I=13\end{array}$ & 13 & $\begin{array}{c}D=1,18^{\prime \prime} \\
I=1,46^{\prime \prime}\end{array}$ & -18 & $\begin{array}{l}D=+1 \\
I=+1,5\end{array}$ \\
\hline Silva Juan Elias & 86 & $\begin{array}{l}D=13 \\
I=15\end{array}$ & 12 & $\begin{array}{l}D=0^{\prime \prime} \\
I=0^{\prime \prime}\end{array}$ & -42 & $\begin{array}{c}D=-3,3 \\
I=-7\end{array}$ \\
\hline Villavicencio Leopoldo & 88 & $\begin{array}{l}D=0 \\
I=11\end{array}$ & 12 & $\begin{array}{l}D=0^{\prime \prime} \\
I=11^{\prime \prime}\end{array}$ & -13 & $\begin{array}{l}D=+3 \\
I=+2,5\end{array}$ \\
\hline Ximenez Luz & 73 & $\begin{array}{l}D=19 \\
I=15\end{array}$ & 13 & $\begin{array}{l}D=0^{\prime \prime} \\
I=0^{\prime \prime}\end{array}$ & -36 & $\begin{array}{l}D=+3 \\
I=+2,5\end{array}$ \\
\hline
\end{tabular}

Fuente: Elaboración propia.

Para el estudio comparativo, se valoraron los test físicos de: Flexión de codo, sentadilla, equilibrio estático, flexibilidad del tren superior, y flexibilidad del tren inferior, a un totalde 
6 personas del "Hogar del Adulto Mayor San Ignacio de Loyola", tal como se muestra en la Tabla 1, dónde los literales indicados como "D" e "I", se relacionan con los resultados por miembros de: Derecha e Izquierda respectivamente. Obteniendo valores significativos de cada participante.

Tabla 2. Hogar de Ancianos Mi Amigo Divino

\begin{tabular}{|c|c|c|c|c|c|c|}
\hline NOMBRES & EDAD & $\begin{array}{l}\text { FLEXION } \\
\text { CODO }\end{array}$ & SENTADILLA(30") & $\begin{array}{c}\text { EQUILIBRIO } \\
\text { ESTATICO }\end{array}$ & $\begin{array}{l}\text { FLEXIBILIDAD } \\
\text { TREN } \\
\text { SUPERIOR }\end{array}$ & $\begin{array}{l}\text { FLEXIBILIDAD } \\
\text { TREN } \\
\text { INFERIOR }\end{array}$ \\
\hline \multirow{2}{*}{$\begin{array}{l}\text { Acuña Sosa María } \\
\text { Magdalena }\end{array}$} & \multirow{2}{*}{63} & $D=8$ & \multirow{2}{*}{10} & $D=2^{\prime \prime}$ & \multirow{2}{*}{-14} & $D=-1$ \\
\hline & & $I=7$ & & $\mathrm{I}=1^{\prime \prime}$ & & $I=-2$ \\
\hline \multirow{2}{*}{ Cuña Martha } & \multirow[b]{2}{*}{57} & $D=12$ & \multirow[b]{2}{*}{12} & $D=2^{\prime \prime}$ & \multirow[b]{2}{*}{-4} & $D=+1$ \\
\hline & & $I=13$ & & $\mathrm{I}=1^{\prime \prime}$ & & $I=0$ \\
\hline \multirow{2}{*}{$\begin{array}{c}\text { Galindo Vargas María } \\
\text { Eugenia }\end{array}$} & \multirow{2}{*}{78} & $D=10$ & \multirow{2}{*}{8} & $D=2^{\prime \prime}$ & \multirow{2}{*}{-15} & $D=+1$ \\
\hline & & $I=0$ & & $I=3^{\prime \prime}$ & & $I=-1$ \\
\hline \multirow{2}{*}{ Ojeda Delia } & \multirow{2}{*}{75} & $\mathrm{D}=10$ & \multirow{2}{*}{10} & $D=5^{\prime \prime}$ & \multirow{2}{*}{-16} & $D=+1$ \\
\hline & & $\mathrm{I}=13$ & & $I=3^{\prime \prime}$ & & $I=-3$ \\
\hline
\end{tabular}

Fuente: Elaboración propia.

Del mismo modo, y bajo el mismo formato de la Tabla 1, la Tabla 2 refleja los resultados obtenidos del "Hogar de Ancianos Mi Amigo Divino", con un total de 4 participantes.

\section{Resultados IPAQ corto.}

Tabla 3. Hogar del Adulto Mayor San Ignacio de Loyola

\begin{tabular}{|c|c|c|c|c|c|c|c|}
\hline SEXO & EDAD & $\begin{array}{l}\text { ¿DURANTE LOS } \\
\text { ÚLTIMOS } 7 \\
\text { DíAS CUANTOS } \\
\text { REALIZO } \\
\text { ACTIVIDAD } \\
\text { MODERADA? }\end{array}$ & $\begin{array}{l}\text { ¿USUALMENTE, } \\
\text { CUANTO } \\
\text { TIEMPO } \\
\text { DEDICA USTED } \\
\text { EN UNO DE } \\
\text { ESOS DÍAS? }\end{array}$ & $\begin{array}{l}\text { ¿DURANTE LOS } \\
\text { ÚLTIMOS } 7 \\
\text { DíAS, } \\
\text { CUANTOS } \\
\text { CAMINO } \\
\text { USTED POR LO } \\
\text { MENOS } 10 \\
\text { MINUTOS } \\
\text { CONTINUOS? }\end{array}$ & $\begin{array}{l}\text { ¿USUALMENTE, } \\
\text { CUANTO } \\
\text { TIEMPO GASTÓ } \\
\text { USTED EN UNO } \\
\text { DE ESOS DÍAS } \\
\text { CAMINANDO? }\end{array}$ & $\begin{array}{l}\text { ¿DURANTE LOS } \\
\text { ÚLTIMOS } 7 \\
\text { DÍAS, CUANTO } \\
\text { TIEMPO } \\
\text { PERMANECIÓ } \\
\text { SENTADO(A) } \\
\text { EN UN DÍA? }\end{array}$ & $\begin{array}{l}\text { NIVEL DE } \\
\text { ACTIVIDAD } \\
\text { FÍSICA }\end{array}$ \\
\hline Masculino & 85 & 7 & 30 & 3 & 180 & 120 & Moderado \\
\hline Femenino & 90 & 4 & 20 & 7 & 120 & 300 & Alta \\
\hline Masculino & 99 & 3 & 25 & 7 & 30 & 180 & Moderado \\
\hline Femenino & 93 & 7 & 15 & 7 & 15 & 240 & Moderado \\
\hline Masculino & 85 & 4 & 20 & 7 & 10 & 240 & Baja \\
\hline Femenino & 94 & 7 & 120 & 7 & 180 & 30 & Alta \\
\hline Masculino & 85 & 0 & 0 & 7 & 10 & 240 & Moderada \\
\hline
\end{tabular}

Fuente: Elaboración propia. 
En cuyos casos dónde la aplicación del test físico no fue una opción dadas las capacidades físicas del adulto mayor participante, se aplicó la Encuesta Internacional de Actividad Física (IPAQ). La Tabla 3 refleja el resultado obtenido de las respuestas de 7 encuestados del Hogar del Adulto Mayor San Ignacio de Loyola, describiendo el valor otorgado a cada una de las preguntas que el cuestionario exige, dónde, además, en la última columna se valora el resultado del Nivel de Actividad Física, siendo dividido en: Alto, moderado y baja, según la respuesta de cada adulto mayor participante.

Tabla 4. Hogar de Ancianos Mi Amigo Divino

\begin{tabular}{|c|c|c|c|c|c|c|c|}
\hline SEXO & EDAD & $\begin{array}{l}\text { ¿DURANTE LOS } \\
\text { ÚLTIMOS } 7 \\
\text { DÍAS CUANTOS } \\
\text { REALIZO } \\
\text { ACTIVIDAD } \\
\text { MODERADA? }\end{array}$ & $\begin{array}{l}\text { ¿USUALMENTE, } \\
\text { CUANTO TIEMPO } \\
\text { DEDICA USTED } \\
\text { EN UNO DE ESOS } \\
\text { DIAS? }\end{array}$ & $\begin{array}{l}\text { ¿DURANTE } \\
\text { LOS ÚLTIMOS } \\
7 \text { DIAS, } \\
\text { CUANTOS } \\
\text { CAMINO } \\
\text { USTED POR } \\
\text { LO MENOS } 10 \\
\text { MINUTOS } \\
\text { CONTINUOS? }\end{array}$ & $\begin{array}{l}\text { ¿USUALMENTE, } \\
\text { CUANTO TIEMPO } \\
\text { GASTÓ USTED EN } \\
\text { UNO DE ESOS } \\
\text { DIAS } \\
\text { CAMINANDO? }\end{array}$ & $\begin{array}{l}\text { ¿DURANTE LOS } \\
\text { ÚLTIMOS } 7 \\
\text { DÍAS, CUANTO } \\
\text { TIEMPO } \\
\text { PERMANECIÓ } \\
\text { SENTADO(A) } \\
\text { EN UN DÍA? }\end{array}$ & $\begin{array}{c}\text { NIVEL DE } \\
\text { ACTIVIDAD FISICA }\end{array}$ \\
\hline Femenino & 82 & 3 & 15 & 3 & 15 & 300 & Baja \\
\hline Femenino & 83 & 7 & 10 & 7 & 15 & 300 & Moderada \\
\hline Femenino & 80 & 0 & 0 & 7 & 60 & 180 & Moderada \\
\hline Femenino & 99 & 7 & 5 & 7 & 10 & 600 & Baja \\
\hline Femenino & 97 & 7 & 5 & 7 & 15 & 600 & Baja \\
\hline Femenino & 99 & 7 & 15 & 7 & 10 & 600 & Moderado \\
\hline Femenino & 85 & 0 & 0 & 7 & 540 & 120 & Alta \\
\hline Femenino & 81 & 0 & 0 & 7 & 20 & 240 & Baja \\
\hline Masculino & 100 & 0 & 0 & 7 & 5 & 600 & Baja \\
\hline Femenino & 87 & 0 & 0 & 0 & 0 & 600 & Baja \\
\hline Masculino & 98 & 7 & 20 & 7 & 15 & 600 & Moderada \\
\hline
\end{tabular}

Fuente: Elaboración propia.

Del mismo modo, y bajo el formato de la Tabla 3, la Tabla 4 refleja los datos tomados a un grupo de 11 adultos mayores encuestados, esta vez del Hogar de Ancianos Mi Amigo Divino.

Tabla 5. Flexión de codo derecho

Rangos

\begin{tabular}{|ll|r|r|r|}
\hline & Pariado & N & \multicolumn{1}{c|}{$\begin{array}{c}\text { Rango } \\
\text { promedio }\end{array}$} & $\begin{array}{c}\text { Suma de } \\
\text { rangos }\end{array}$ \\
\hline Flexioncodo1 & Loyola & 6 & 6,17 & 37,00 \\
& A.divino & 4 & 4,50 & 18,00 \\
& Total & 10 & & \\
\hline
\end{tabular}


Estadísticos de contraste ${ }^{a}$

\begin{tabular}{|l|r|}
\hline & Flexioncodo1 \\
\hline U de Mann-Whitney & 8,000 \\
W de Wilcoxon & 18,000 \\
$Z$ &,- 861 \\
Sig. asintót. (bilateral) &, 389 \\
Sig. exacta [2*(Sig. unilateral)] &, $476^{\mathrm{b}}$ \\
\hline
\end{tabular}

a. Variable de agrupación: Pariado

b. No corregidos para los empates.

Fuente: Elaboración propia.

En la Tabla 5 se describe la valoración de la flexión de codo derecho, dándonos como resultado que no existe una diferencia significativa al encontrarse por encima del 0,05 .

Tabla 6. Flexión de codo izquierdo

Rangos

\begin{tabular}{|ll|r|r|r|}
\hline & Pariado & $\mathrm{N}$ & \multicolumn{1}{c|}{$\begin{array}{c}\text { Rango } \\
\text { promedio }\end{array}$} & $\begin{array}{c}\text { Suma de } \\
\text { rangos }\end{array}$ \\
\hline Flexioncodolzquierdo & Loyola & 6 & 6,25 & 37,50 \\
& A.divino & 4 & 4,38 & 17,50 \\
& Total & 10 & & \\
\hline
\end{tabular}

Estadísticos de contraste ${ }^{a}$

\begin{tabular}{|l|r|}
\hline & $\begin{array}{r}\text { Flexioncodolz } \\
\text { quierdo }\end{array}$ \\
\hline U de Mann-Whitney & 7,500 \\
W de Wilcoxon & 17,500 \\
$Z$ &,- 996 \\
Sig. asintót. (bilateral) &, 319 \\
Sig. exacta [2*(Sig. &, $352^{\mathrm{b}}$ \\
unilateral)] & \\
\hline
\end{tabular}

a. Variable de agrupación: Pariado

b. No corregidos para los empates.

Fuente: Elaboración propia.

En la Tabla 6 se describe la valoración de la flexión de codo izquierdo, dándonos como resultado que no existe una diferencia significativa al encontrarse por encima del 0,05. 
Tabla 7 - Sentadilla

Rangos

\begin{tabular}{|c|c|c|c|c|}
\hline & Pariado & $N$ & $\begin{array}{c}\text { Rango } \\
\text { promedio }\end{array}$ & $\begin{array}{c}\text { Suma de } \\
\text { rangos }\end{array}$ \\
\hline \multirow[t]{3}{*}{ sentadilla } & Loyola & 6 & 6,67 & 40,00 \\
\hline & A.divino & 4 & 3,75 & 15,00 \\
\hline & Total & 10 & & \\
\hline
\end{tabular}

Estadísticos de contraste ${ }^{a}$

\begin{tabular}{|l|r|}
\hline & $\begin{array}{r}\text { Equilibrioestá } \\
\text { ticoderecha }\end{array}$ \\
\hline U de Mann-Whitney & 3,000 \\
W de Wilcoxon & 24,000 \\
$Z$ & $-2,006$ \\
Sig. asintót. (bilateral) &, 045 \\
Sig. exacta [2*(Sig. &, $067^{\mathrm{b}}$ \\
unilateral)] & \\
\hline
\end{tabular}

a. Variable de agrupación: Pariado

b. No corregidos para los empates.

Fuente: Elaboración propia.

En la Tabla 7 se describe la valoración de la sentadilla, dándonos como resultado que no existe una diferencia significativa al encontrarse por encima del 0,05.

Tabla 8. Equilibrio estático derecha

Rangos

\begin{tabular}{|ll|r|r|r|}
\hline & Pariado & N & \multicolumn{1}{c|}{$\begin{array}{c}\text { Rango } \\
\text { promedio }\end{array}$} & $\begin{array}{c}\text { Suma de } \\
\text { rangos }\end{array}$ \\
\hline Equilibrioestáticoderecha & Loyola & 6 & 4,00 & 24,00 \\
& A.divino & 4 & 7,75 & 31,00 \\
& Total & 10 & & \\
\hline
\end{tabular}

Estadísticos de contraste ${ }^{\mathrm{a}}$

\begin{tabular}{|l|r|}
\hline & $\begin{array}{r}\text { Equilibrioestá } \\
\text { ticoderecha }\end{array}$ \\
\hline U de Mann-Whitney & 3,000 \\
W de Wilcoxon & 24,000 \\
$Z$ & $-2,006$ \\
Sig. asintót. (bilateral) &, 045 \\
Sig. exacta [2*(Sig. &, $067^{\mathrm{b}}$ \\
unilateral)] & \\
\hline
\end{tabular}

a. Variable de agrupación: Pariado

b. No corregidos para los empates.

Fuente: Elaboración propia. 
En la Tabla 8se describe la valoración del equilibrio estático derecho, dándonos como resultado que no existe una diferencia significativa al encontrarse por encima del 0,05.

Tabla 9. Equilibrio estático izquierda

\begin{tabular}{|ll|r|r|r|}
\hline \multicolumn{1}{|c|}{ Rangos } \\
\hline & Pariado & N & \multicolumn{1}{c|}{$\begin{array}{c}\text { Rango } \\
\text { promedio }\end{array}$} & $\begin{array}{c}\text { Suma de } \\
\text { rangos }\end{array}$ \\
\hline Equilibrioestáticoizquierd & Loyola & 6 & 4,83 & 29,00 \\
a & A.divino & 4 & 6,50 & 26,00 \\
& Total & 10 & & \\
\hline
\end{tabular}

\begin{tabular}{|l|r|}
\hline & $\begin{array}{c}\text { Equilibrioestá } \\
\text { ticoizquierda }\end{array}$ \\
\hline U de Mann-Whitney & 8,000 \\
W de Wilcoxon & 29,000 \\
$Z$ &,- 869 \\
Sig. asintót. (bilateral) &, 385 \\
Sig. exacta [2*(Sig. &, $476^{\text {b }}$ \\
unilateral)] & \\
\hline
\end{tabular}

a. Variable de agrupación: Pariado

b. No corregidos para los empates.

Fuente: Elaboración propia.

En la Tabla 9 se describe la valoración del equilibrio estático izquierdo, dándonos como resultado que no existe una diferencia significativa al encontrarse por encima del 0,05 .

Tabla 10. Flexibilidad del tren superior

Rangos

\begin{tabular}{|ll|r|r|r|}
\hline & Pariado & N & \multicolumn{1}{c|}{$\begin{array}{c}\text { Rango } \\
\text { promedio }\end{array}$} & $\begin{array}{c}\text { Suma de } \\
\text { rangos }\end{array}$ \\
\hline flexibilidadsuperior & Loyola & 6 & 4,50 & 27,00 \\
& A.divino & 4 & 7,00 & 28,00 \\
& Total & 10 & & \\
\hline
\end{tabular}

Estadísticos de contraste ${ }^{a}$

\begin{tabular}{|l|r|}
\hline & $\begin{array}{c}\text { flexibilidadsu } \\
\text { perior }\end{array}$ \\
\hline U de Mann-Whitney & 6,000 \\
W de Wilcoxon & 27,000 \\
$Z$ & $-1,283$ \\
Sig. asintót. (bilateral) &, 199 \\
Sig. exacta [2*(Sig. &, $257^{\mathrm{b}}$ \\
unilateral)] & \\
\hline
\end{tabular}

a. Variable de agrupación: Pariado

b. No corregidos para los empates.

Fuente: Elaboración propia. 
En la Tabla 10 se describe la valoración de la flexibilidad del tren superior, dándonos como resultado que no existe una diferencia significativa al encontrarse por encima del 0,05 .

Tabla 11. Flexibilidad del tren inferior izquierda y derecha

\begin{tabular}{|c|c|c|c|c|}
\hline \multicolumn{5}{|c|}{ Rangos } \\
\hline & Pariado & $\mathrm{N}$ & $\begin{array}{c}\text { Rango } \\
\text { promedio }\end{array}$ & $\begin{array}{c}\text { Suma de } \\
\text { rangos }\end{array}$ \\
\hline \multirow[t]{2}{*}{ flexibilidadinferiorl } & Loyola & 6 & 6,67 & 40,00 \\
\hline & A.divino & 4 & 3,75 & 15,00 \\
\hline
\end{tabular}

Estadísticos de contraste
\begin{tabular}{|l|r|}
\hline \multicolumn{1}{|c|}{${ }^{\mathrm{a}}$} \\
\hline U de Mann-Whitney & $\begin{array}{r}\text { flexibilidadinfe } \\
\text { riorl }\end{array}$ \\
W de Wilcoxon & 5,000 \\
Z & 15,000 \\
Sig. asintót. (bilateral) & $-1,497$ \\
Sig. exacta [2*(Sig. &, 134 \\
unilateral)] &, $171^{\text {b }}$ \\
\hline
\end{tabular}

a. Variable de agrupación: Pariado

b. No corregidos para los empates.

Estadísticos de contraste ${ }^{a}$

\begin{tabular}{|l|r|}
\hline & $\begin{array}{r}\text { flexibilidadinfe } \\
\text { riorl }\end{array}$ \\
\hline U de Mann-Whitney & 5,000 \\
W de Wilcoxon & 15,000 \\
Z & $-1,497$ \\
Sig. asintót. (bilateral) &, 134 \\
Sig. exacta [2*(Sig. &, $171^{\text {b }}$ \\
unilateral)] & \\
\hline
\end{tabular}

a. Variable de agrupación: Pariado

b. No corregidos para los empates.

En la Tabla 11 se describe la valoración de la flexibilidad del tren inferior, dándonos como resultado que no existe una diferencia significativa al encontrarse por encima del 0,05.

\section{Conclusiones.}

- Los resultados de las valoraciones arrojan datos iniciales que corresponden directamente al tipo de actividad que promueven los centros de tratamiento al adulto mayor de forma independiente, no encontrándose una diferencia significativa entre las valoraciones. 


\section{Referencias bibliográficas.}

Casanueva, E., Kaufer, M., Pérez, A. B., \& Arroyo, P. (2008). Nutriología Médica. México D.F.: Médica Panamericana.

Cervera, V. O. (1999). Entrenamiento de fuerza y explosividad para la actividad física y el deporte de competición (Segunda ed.). Barcelona: Inde.

Cevallos, O. (2012). Actividad Física en el Adulto Mayor. Colombia: El manual moderno.

Durán, L. R. (2013). La Vejez en Movimiento un enfoque Integral. Buenos Aires: Dunken.

Gil, A. (2010). tratado de nutrición (segunda ed.). Buenos Aires: Panamericana.

Heredia, J., Donate, F., Medrano, I., Riera, M., \& Mata, F. (2011). Entrenamiento de flexibilidad para la salud. Sevilla: Wanceulen.

Maranzano, S. (2013). Actividad Física para la tercera edad (Séptima ed.). Buenos Aires: dunken.

Martin, D. (2001). Manual de metodología del entrenamiento deportivo. Barcelona: Paidotribo.

Riedel, M. (2000). El proceso de envejecimiento (Tercera ed.). Madrid: Morata.

Rikly, R., Jones, J., (1999) Development and validation of a functional fitness test for community residing older adults

Smith, M. L. (2009). Health literacy and weight-related behaviors among.Kansas: ProQuest..

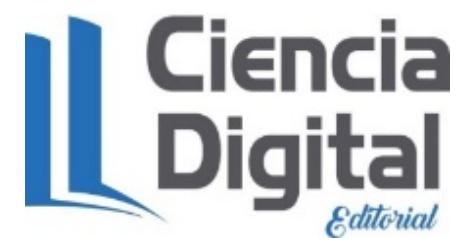




\section{Para citar el artículo indexado}

Coca, O. R., Flores, M. C., Gómez Nicolalde, R. V., \& Zamora Parra, X. A. (2020). Estudio comparativo sobre las capacidades físicas del adulto mayor. AlfaPublicaciones, 2(4), 38-50. https://doi.org/10.33262/ap.v2i4.13

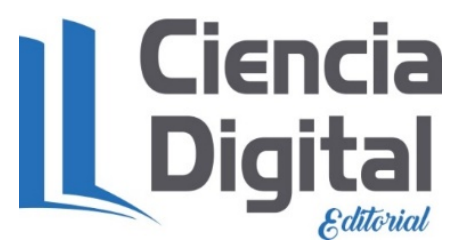

El artículo que se publica es de exclusiva responsabilidad de los autores y no necesariamente reflejan el pensamiento de la Revista Alpha Publicaciones.

El artículo queda en propiedad de la revista y, por tanto, su publicación parcial y/o total en otro medio tiene que ser autorizado por el director de la Revista Alpha Publicaciones.
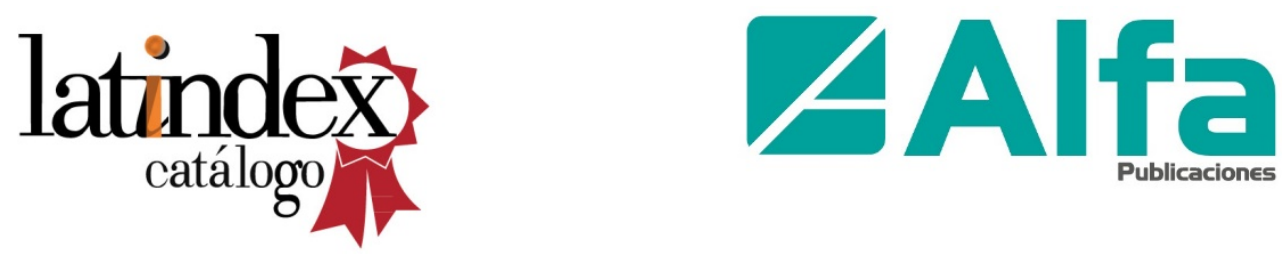